\title{
Avascular Necrosis of Humeral Head after Thalidomide Use: A Report of Two Cases
}

\author{
Ahmad Rezaeian* \\ Department of Otorhinolaryngology, Iran \\ *Corresponding author: Ahmad Rezaeian, Department of Otorhinolaryngology, Iran
}

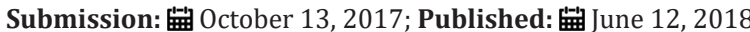

\begin{abstract}
Background: Thalidomide is an immunomodulatory drug which is used for treatment of certain cancers such as multiple myeloma. Multiple myeloma (MM) is a cancer that creates a specific type of white blood cell called plasma cell and accumulates these cancerous cells in bone marrow. To our knowledge, there is no report of avascular necrosis of humeral head after thalidomide use; hence, the aim of present study was to report two cases of avascular necrosis of humeral head after thalidomide use in MM patients.
\end{abstract}

Case reports: Twenty five patients with history of MM were evaluated for the adverse effects of thalidomide from 2008 to 2014 at Department of Orthopedics, Isfahan University of Medical Sciences-Isfahan- Iran. All cases were assessed by X-ray and bone scan.

Conclusion: Out of 25 , two cases were diagnosed with avascular necrosis of humeral head with pain in shoulders. It seems that thalidomide can cause necrosis in humeral head.

Keywords: Thalidomide; Multiple myeloma; Necrosis; Humeral bone

\section{Introduction}

Multiple Myeloma (MM) is a disabling cancer that is part of a spectrum of diseases ranging from monoclonal gammopathy of unknown significance to plasma cell leukemia. MM is characterized by several features, including low blood counts, bone and calcium problems, infections, kidney problems, monoclonal gammopathy, light chain amyloidosis and solitary plasmacytoma [1]. Thalidomide was first introduced for patients with refractory multiple myeloma. Due to the promising results achieved in these patients, the drug was subsequently used in the earlier stages of the disease [2]. In this paper, we review the available clinical data regarding the adverse effects of thalidomide in the patients with MM.

\section{Case Report}

\section{Case one}

Presentation: A 63-year-old man was referred to the Orthopedics department by an oncologist owing to right upper limb pain. He had been diagnosed with MM 18 months ago and had been under treatment by thalidomide $(200 \mathrm{mg} /$ day) for 16 months. The patient used acetaminophen (pro re nate) for the shoulder and joint pain. He had a remarkable medical history, including peptic ulcer disease and gastrointestinal bleeding and was under treatment by protein pump inhibitor (Omiperazol 20mg/day) and histamine receptor blocker (Famotidine $10 \mathrm{mg} /$ day). There was no report and history of sickle cell anemia, gaucher disease, alcohol use, hypercoagulopathy and use of bisphosphonate. The patient did not use any corticosteroids, too. He suffered from increasing shoulder pain and reported having a long period of suffering. Pain was more in the right shoulder, particularly in the range of motion. During the increasing pain, a limited range of motion in daily activities was observed. Limitations extended over months.

Clinical evaluation: Clinical examinations showed right shoulder muscular atrophy. In all range of motions, including flexion, abduction, rotation limitation and pain on was remarkable.

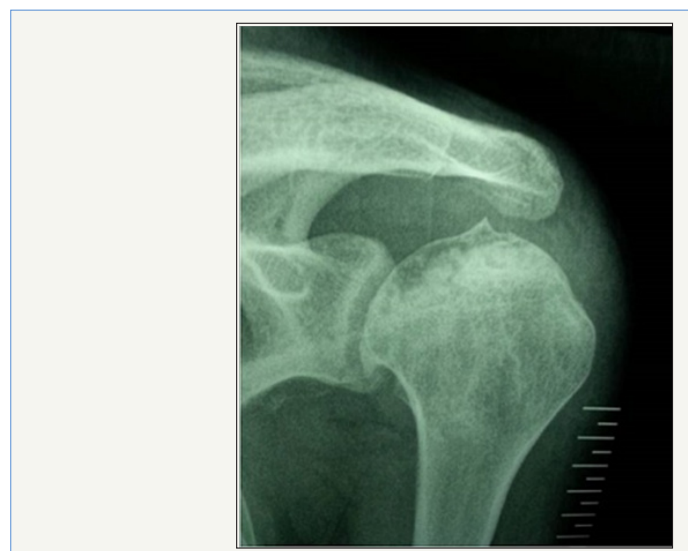

Figure 1: True anterior-posterior (AP) view in case 1: subchondral sclerosis and radiolucent area in humeral head.

Radiographic evaluation: Standard radiological diagnoses include conventional x-ray images. True anterior-posterior (AP) view (Figure 1) showed subchondral sclerosis and a radiolucent 
area in humeral head. Also, there were some degenerative changes in the articular surface of humeral head. MRI determined the avascular necrosis of humeral head (Figure 2).

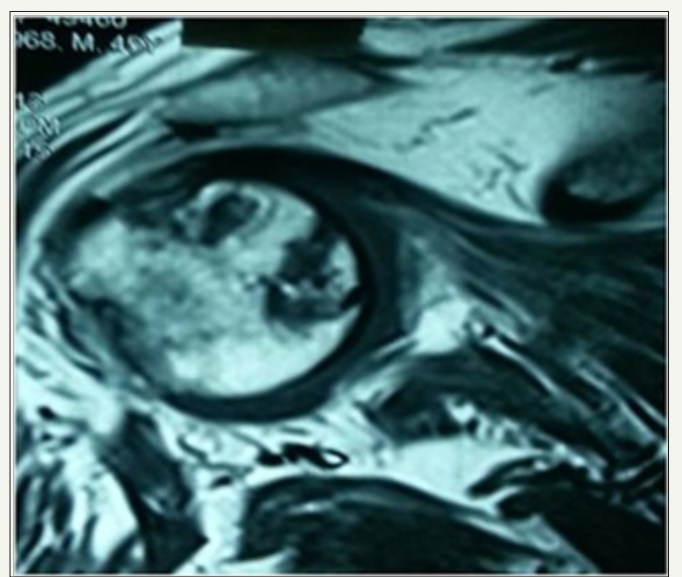

Figure 2: MRI view in case 1: the avascular necrosis of humeral head.

\section{Case two}

Presentation: A 59-year-old female was referred to the Orthopedics Department by an oncologist owing to left upper limb and lumbar pain. He had been diagnosed with MM 13 months ago and had been under treatment by thalidomide $(200 \mathrm{mg} /$ day) for 13 months. The patient used acetaminophen (PRN) for the shoulder and joint pain. She had a remarkable medical history, including diabetic mellitus, for which she used metformin $500 \mathrm{mg} /$ day. There was no report and history of sickle cell anemia, gaucher disease, alcohol use, hypercoagulopathy and use of bisphosphonate. The patient did not use any corticosteroids, too. She suffered from increasing lumbar and shoulder pain and reported having had this condition since three months ago. Pain was more in the left shoulder, particularly in the range of motion. Owing to left shoulder pain, the patient had limited mobility and loss of function in daily activities.

Clinical evaluation: Clinical examinations showed left shoulder muscular atrophy. In all range of motions, including flexion, abduction, rotation limitation and pain on was remarkable. Also, tenderness was noted in lumbar spine examination.

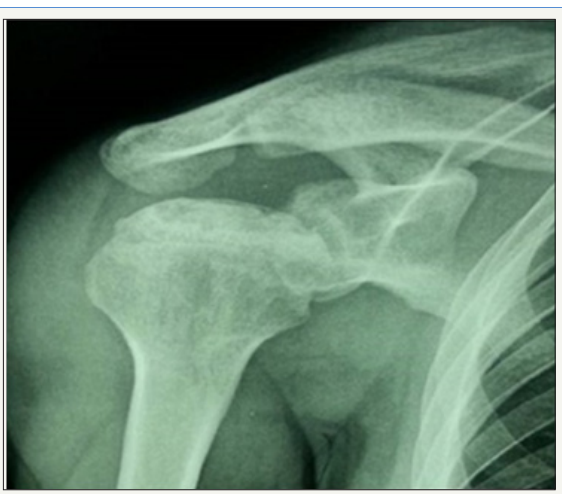

Figure 3: True anterior-posterior (AP) view in case 2: the subchondral sclerosis and radiolucent area in humeral head.

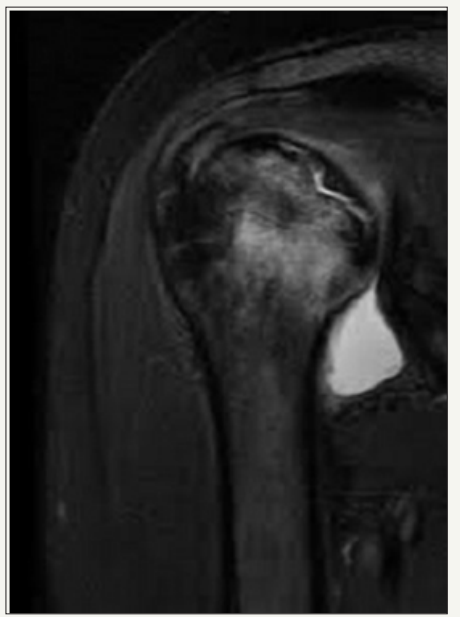

Figure 4: MRI view in case 2: the avascular necrosis of humeral head.

Radiographic evaluation: Standard radiological diagnoses include conventional x-ray images. True anterior-posterior (AP) view (Figure 3) revealed subchondral sclerosis and a radiolucent area in humeral head. Also, there were some degenerative changes in the articular surface of humeral head. MRI determined the avascular necrosis of humeral head (Figure 4).

\section{Discussion}

In this study, we found avascular necrosis in two Iranian patients with multiple myeloma disease who were treated with thalidomide (200mg/day) for more than 10 months. Therefore, thalidomide may cause necrosis in the articular surface, although this case report is the first study reporting avascular necrosis in treatment with thalidomide in multiple myeloma patients. Thalidomide is an immunomodulatory and anti-inflammatory drug which stops neoangiogenesis in malignant tumors, although its mechanism is still unknown $[3,4]$. We have this hypothesis that thalidomide prevents neoangiogenesis in cartilage; therefore, it may be caused by avascular necrosis. Other studies have shown that thalidomide is an endogenous metabolite of estradiol and 2-methoxyestradiol and suppresses disease in collagen-induced arthritis by exerting antiangiogenic effects $[5,6]$ In the study by Song et al. [7], thalidomide exacerbated zoledronate-induced firststage BRONJ (bisphosphonate-related osteonecrosis of the jaws) and caused osteonecrosis of the jaw and inhibition of angiogenesis. Also, thalidomide has teratogenic effects and causes orthopedic malformations. In the past, thalidomide was used for sedation in pregnant woman; therefore, the children were born with many musculoskeletal malformations. Musculoskeletal presentations of this children included spinal defects such as block vertebrae, spondylolysis, scoliosis, malformations of intervertebral disc and dysgenesia of sacrum [8-11]. In the study by Panousis evaluating thalidomide effect on musculoskeletal injury in an experimental model, thalidomide prolonged the survival of experimental models with musculoskeletal injury by preventing mononuclear apoptosis. Therefore, thalidomide can be used for management of severe traumas [12]. Thalidomide has several adverse effect 
on the patients with multiple myeloma, but the most common complication is toxic neuropathy. Other side effect is less common and is controlled easily by drugs [13]. In the present study, the patients were treated with a low dose of thalidomide. More adverse effects of thalidomide are also dose-dependent. Offidani et al. [14] showed an association between thalidomide dose and side effects in multiple myeloma; therefore, the most common side effects such as constipation, somnolence and fatigue were not dose-dependent. However, peripheral neuropathy was dose-dependent. Also, a daily dose of thalidomide (150mg) had minimal side effects. The study by Talamo et al. [15] had a opposite result with our study, they concluded that avascular necrosis is a rare complication in multiple myeloma so male sex, dexamethasone dose and younger age increased risk of avascular necrosis and didn't have relation between thalidomide and avascular necrosis.

\section{Conclusion}

In conclusion, the other adverse effects of thalidomide are rare but may occur. Moreover, musculoskeletal side effects may occur as a result of using thalidomide in multiple myeloma, but in a dosedependent manner. Therefore, there was no report of avascular necrosis in Iranian multiple myeloma patients as a result of using thalidomide $(200 \mathrm{mg} /$ day), but we found two cases with avascular necrosis in using thalidomide possibly due to antiangiogenic effects on articular cartilage. Hence, future studies are required to confirm our findings.

\section{References}

1. Stamo A, Grigor'eva V (2016) Clinical characteristics of pain syndrome in patients with multiple myeloma. Zhurnal nevrologii i psikhiatrii imeni SS Korsakova 116(10): 11.

2. Musto P, Anderson K, Attal M, Richardson P, Badros A, et al. (2016) Second primary malignancies in multiple myeloma: an overview and IMWG consensus. Ann Oncol 28(2): 228-245.

3. Bertolini F, Mingrone W, Alietti A, Ferrucci P, Cocorocchio E, et al. (2001) Thalidomide in multiple myeloma, myelodysplastic syndromes and histiocytosis. Analysis of clinical results and of surrogate angiogenesis markers. Ann Oncol 12(7): 987-990.
4. Zangari M, Elice F, Tricot $G$ (2005) Immunomodulatory drugs in multiple myeloma. Expert Rev Hematol 6(1): 69-82.

5. Josefsson E, Tarkowski A (1997) Suppression of type II collagen-induced arthritis by the endogenous estrogen metabolite 2-methoxyestradiol. Arthritis \& Rheumatism 40(1): 154-163.

6. Oliver S, Cheng T, Banquerigo M, Brahn E (1998) The effect of thalidomide and 2 analogs on collagen induced arthritis. The Journal of rheumatology 25(5): 964-969.

7. Song Z, Dong W, Yin L, Liu J, Sun H, et al. (2015) Effect of thalidomide on development of bisphosphonate-related osteonecrosis of the jaws in rats. Journal of Southern Medical University 35(8): 1084-1089.

8. Lenz W, Knapp K (1962b) Thalidomide embryopathy. Arch Environm Health 5: 100-105.

9. Brent RL, Holmes LB (1988) Clinical and basic science lessons from the thalidomide tragedy: what have we learned about the causes of limb defects? Teratology 38(3): 241-251.

10. Ruffing L (1980) The spine in the thalidomide-embryopathy. Fortschritte der Medizin 98(11): 405-409.

11. Edwards D, Nichols P (1977) The spinal abnormalities in thaltdomide embryopathy. Acta Orthop Scand 48(3): 273-276.

12. Panousis K, Nikolaou VS, Tsaganos T, Lallos S, Bourboulis EJG, et al. (2014) Thalidomide prolongs survival after experimental musculoskeletal injury, through an effect on mononuclear apoptosis. Journal of Surgical Research 188(1): 198-205.

13. Novosad O, Kriachok I, Kadnikova T, Tytorenko I, Sychova T, et al. (2009) Experience of using thalidomide in the treatment of patients with multiple myeloma. Lik Sprava (3-4): 79-86.

14. Offidani M, Corvatta L, Marconi M, Malerba L, Mele A, et al. (2004) Common and rare side-effects of low-dose thalidomide in multiple myeloma: focus on the dose-minimizing peripheral neuropathy. European journal of haematology 72(6): 403-409.

15. Talamo G, Angtuaco E, Walker RC, Dong L, Miceli MH, et al. (2005) A vascular necrosis of femoral and/or humeral heads in multiple myeloma: results of a prospective study of patients treated with dexamethasonebased regimens and high-dose chemotherapy. Journal of clinical oncology 23(22): 5217-5223.
Creative Commons Attribution 4.0 International License

For possible submissions Click Here

\section{Submit Article}

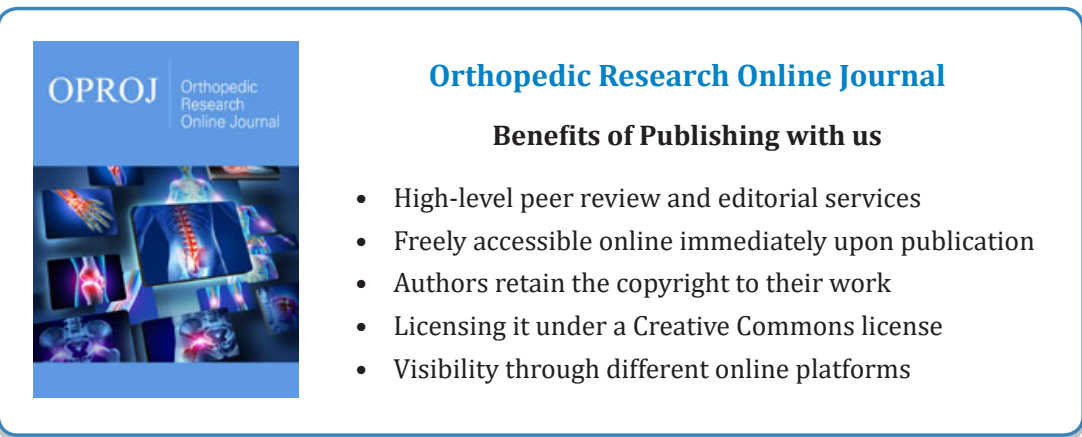

